\title{
Impact of Neoadjuvant Sunitinib Treatment on Tumour Thrombi in the Inferior Vena Cava in Metastatic Renal Cell Carcinoma
}

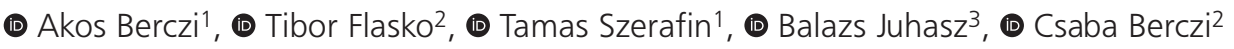 \\ 1 Debrecen University Faculty of Medicine, Department of Cardiac Surgery, Debrecen, Hungary \\ 2 Debrecen University Faculty of Medicine, Department of Urology, Debrecen, Hungary \\ 3 Debrecen University Faculty of Medicine, Department of Oncology, Debrecen, Hungary
}

\begin{abstract}
The authors report cases of metastatic renal cell carcinoma with level III-IV tumour thrombi in the inferior vena cava (IVC). Cases were treated with three courses of neoadjuvant sunitinib to reduce the thrombus level before surgery.

Nephrectomy and tumour thrombectomy were performed, and sunitinib treatment continued after the operation.

Cases 1 and 3 showed regression of lung metastases, but the size of the primary renal tumour and thrombus remained the same. The progression-free survival of the cases was 35 months and 24 months, respectively. In case 2, the primary renal tumour, metastases and thrombus showed regression. The upper limit of the thrombus decreased by $3 \mathrm{~cm}$. In this case, the progression-free survival was 15 months, and the cancer-specific survival was 18 months.

The neoadjuvant sunitinib treatment had a limited effect on downsizing the extent of tumour thrombi in the IVC.

Keywords: Inferior vena cava, neoadjuvant sunitinib, metastatic renal cancer, radical nephrectomy, tumour thrombus
\end{abstract}

\section{Introduction}

Progression of renal cancers into the inferior vena cava (IVC) is relatively rare and is sometimes associated with presence of distant metastases. The conventional treatment for metastatic renal cancer with a tumour thrombus in the IVC is radical nephrectomy with concomitant tumour thrombectomy, followed by targeted molecular therapy. Currently, there are relatively limited publicised data on the impact of neoadjuvant sunitinib therapy on tumour thrombi in the IVC $(1,2,3,4,5)$.

\section{Case Presentation}

We report a series of patients who received neoadjuvant sunitinib treatment for metastatic renal cell carcinoma with a tumour thrombus in IVC. Tumour thrombus levels were determined according to Novick's classification. The tumours were in the intermediate risk group based on Memorial Sloan Kettering Cancer Center criteria. Patients received three courses of neoadjuvant sunitinib treatment, $50 \mathrm{mg} /$ day for 4 weeks followed by a 2-week break before surgery. Afterward sunitinib treatment, the patients underwent abdominal and chest computed tomography (CT) exams and were evaluated according to the response evaluation criteria in solid tumours (RECIST) 1.1 criteria.

\section{Case 1}

A 66 year old male patient had macroscopic haematuria and an abdominal ultrasound described an $8 \mathrm{~cm}$ tumour in the left kidney. Abdominal and chest CT exams showed a suprahepatic tumour thrombus in the IVC (level IV), multiple lung metastases and metastases in the left adrenal gland. Renal biopsy revealed clear cell renal carcinoma. After neoadjuvant sunitinib treatment, the CT control showed a regression of lung metastases, but the size of the primary renal tumour did not change. The upper level of the tumour thrombus also did not decrease (Figure 1) (Table 1).

Radical nephrectomy and tumour thrombectomy were performed and histologic analysis revealed grade 2 clear cell carcinoma with $\mathrm{pT} 3 \mathrm{c}, \mathrm{pN} 0, \mathrm{pM} 1$ stage.

After the surgery, sunitinib therapy was continued according to the protocol with follow-up CT exams performed every two cycles. The actual progression-free survival (PFS) of the patient was 35 months.

Cite this article as: Berczi A, Flasko T, Szerafin T, Juhasz B, Berczi C. Impact of Neoadjuvant Sunitinib Treatment on Tumour Thrombi in the Inferior Vena Cava in Metastatic Renal Cell Carcinoma. Bull Urooncol 2020;19(3):162-164 


\section{Case 2}

A 50 year old female had a deep vein thrombosis and a routine abdominal ultrasound discovered a $14 \mathrm{~cm}$ tumour in the right kidney. The CT exam revealed a renal tumour with a tumour thrombus extending into the right atrium (level IV), as well as multiple liver and retroperitoneal lymph node metastases. Renal biopsy revealed clear cell renal carcinoma. After neoadjuvant sunitinib treatment, the follow-up CT showed partial regression of both the renal tumour and liver metastases. The upper limit of the thrombus decreased by $3 \mathrm{~cm}$, but it remained a level IV thrombus (Figure 2). Radical nephrectomy was performed, but tumour thrombectomy was unsuccessful. Histology of the resected tumour revealed grade 2 clear cell renal carcinoma with pT3a, pNO stage. Following radical nephrectomy, sunitinib treatment was continued according to the protocol. Fifteen months after the radical nephrectomy, the control CT demonstrated a new liver metastasis, and sunitinib therapy was

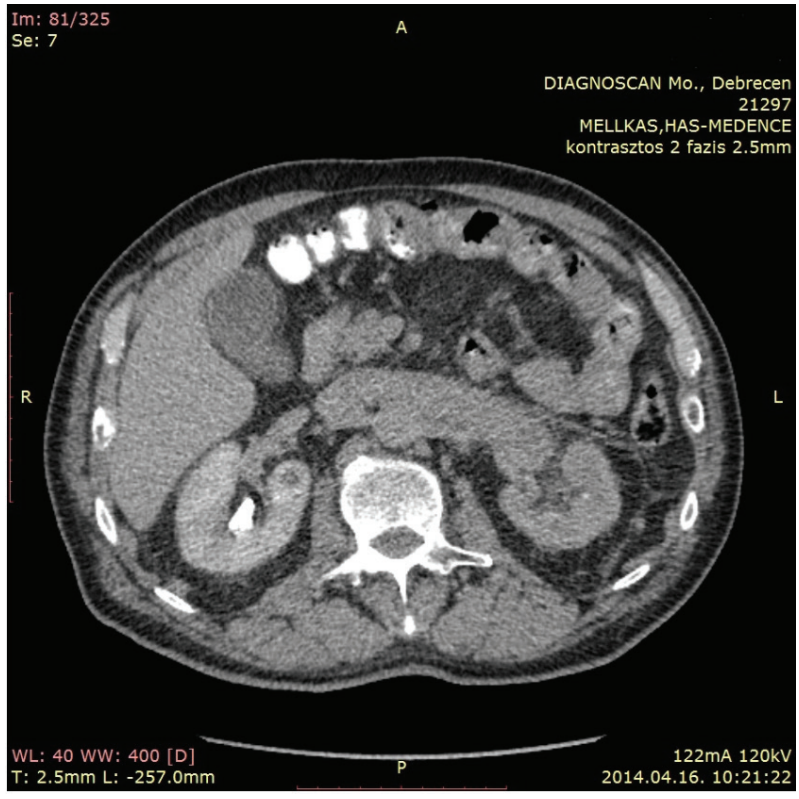

Figure 1. Abdominal computed tomography after the neoadjuvant sunitinib treatment in Case 1 (axial section)

Table 1. Control computed tomography findings after the neoadjuvant sunitinib treatment

\begin{tabular}{|l|l|}
\hline Case $\mathbf{1}$ & Tumour response \\
\hline Renal tumour & Stable \\
\hline Lung metastases & Regression \\
\hline Tumour thrombus & No change \\
\hline Case $\mathbf{2}$ & \multicolumn{2}{|l|}{} \\
\hline Renal tumour & Regression \\
\hline Liver metastases & Regression \\
\hline Tumour thrombus & Decreased by $3 \mathrm{~cm}$ \\
\hline Case 3 & \multicolumn{2}{|l|}{} \\
\hline Renal tumour & Stable (but necrosis) \\
\hline Lung metastases & Regression \\
\hline Tumour thrombus & No change \\
\hline
\end{tabular}

ceased. The patient's PFS was 15 months and the cancer-specific survival was 18 months.

\section{Case 3}

A 52 year old male patient had a visible mass protruding from the upper right abdomen. Ultrasound showed a $22 \mathrm{~cm}$ tumour in the right kidney. Chest and abdominal CT exams showed a renal tumour with multiple lung and retroperitoneal lymph node metastases, as well as an intrahepatic tumour thrombus in the IVC (level III). Renal biopsy revealed clear cell carcinoma. After neoadjuvant sunitinib therapy the follow-up CT showed partial regression of lung metastases. The $\mathrm{CT}$ did not show changes in the bulk size of the renal tumour or the thrombus (Figure 3). Surgery was performed and the histology revealed grade 2 clear cell carcinoma with pT3c, pN2 stage. After the operation, sunitinib therapy was continued according to the protocol. CT

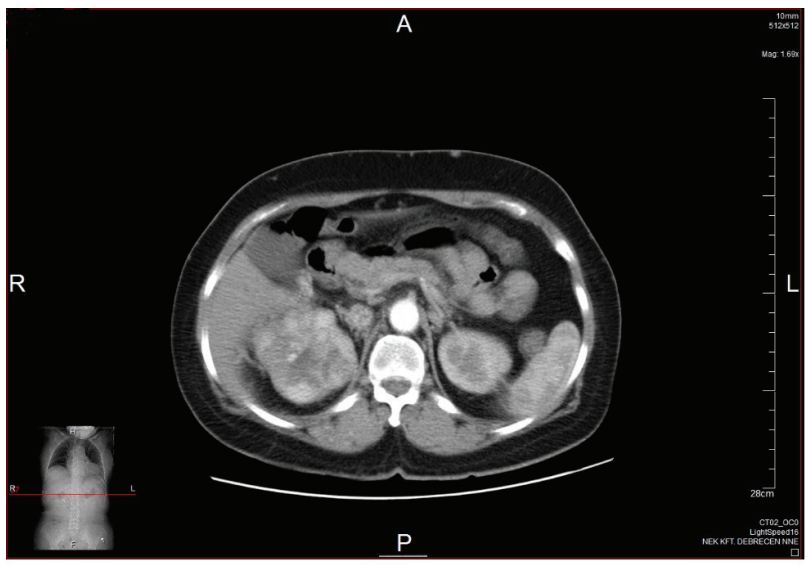

Figure 2. Abdominal computed tomography after the neoadjuvant sunitinib treatment in Case 2 (axial section)

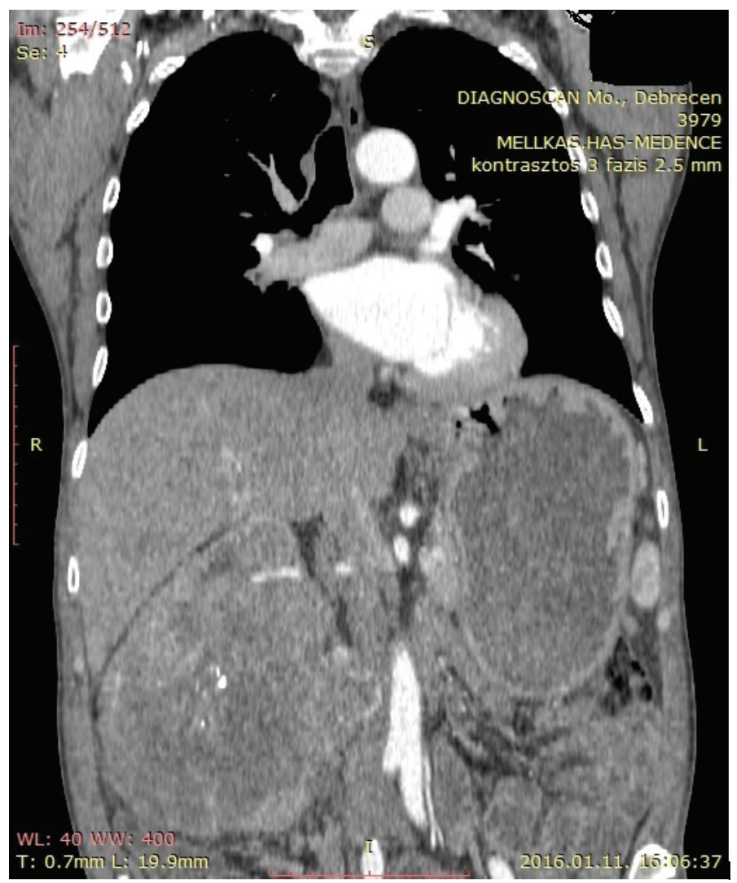

Figure 3. Abdominal computed tomography after the neoadjuvant sunitinib treatment in Case 3 (coronarial section) 
control follow-up exams showed a stable disease on RECIST 1.1, with a PFS of 24 months.

\section{Discussion}

Surgery of renal tumours with IVC thrombi has a relatively high complication rate, ranging from $12 \%$ to $46 \%$. Several physicians have tried to reduce the size of tumour thrombi with administration of neoadjuvant targeted molecular therapy to decrease the rate of complications.

Current literature only provides a few publications in which neoadjuvant therapy has been used to downsize tumour thrombi in the IVC. Generally in these patients, two to four cycles of the targeted therapy were administered before the operation. Of the limited publications, the majority consist of case reports. Some authors described regression of tumour thrombi after neoadjuvant sunitinib therapy in their individual cases $(6,7,8)$. Karakiewicz et al. (7) were the first to report a significant decrease of the thrombus level in the IVC (from level IV to level II) after sunitinib therapy, facilitating easier surgical procedure. Peters et al. (8) described a case in which there was a significant reduction in primary tumour size and metastatic sites, and the IVC thrombus downstaged from level IV to level III according to Novick classification as a result of preoperative sunitinib treatment.

However, in contrast to the mentioned cases, other reports have showed that neoadjuvant therapy was not always successful at downstaging the IVC thrombus level, and progression of the thrombus level was observed $(3,9)$.

Cost et al. (3) studied the efficacy of targeted molecular therapy at decreasing the level of renal cell carcinoma vena cava tumour thrombi in 24 patients. Their data showed that the thrombus level remained unchanged in 21 patients (84\%), decreased in three patients $(12 \%)$ and increased in one patient (4\%) during the treatment. Of the three cases that showed thrombus regression, only one case had enough of an impact to affect the surgical procedure (level IV to III). During the study, the size of the primary tumour increased in 10 patients $(40 \%)$, decreased in $12(48 \%)$, and did not show any change in three (12\%) cases.

Bigot et al. (1) performed a retrospective analysis of 14 patients with renal cell carcinoma with IVC thrombus treated with neoadjuvant sunitinib therapy before the surgery. In the study, six (43\%) patients had a measurable decrease in thrombus size, six $(43 \%)$ had no change, and two (14\%) had an increase. In regard to the thrombus size, 12 patients (85\%) had stable thrombus, one patient (7\%) had a downstage of thrombus level, and one patient (7\%) had an upstage of thrombus level. In the one patient who demonstrated a downstaging of the thrombus (level II to level I), the downstaging was not significant enough to affect the surgical intervention. Follow-up CT exams after the neoadjuvant sunitinib treatment showed that the primary renal tumour remained stable in five patients $(36 \%)$, showed regression in seven patients (50\%), and showed progression in two patients (14\%) (1).

In our cases, regression of the tumour thrombus was detected in only one case, but the tumour thrombus level in that case remained the same (level IV). Neoadjuvant treatment resulted in regression of the distant metastases in all cases, but the size of the primary tumour decreased in only one patient.

\section{Conclusion}

Effective downstaging of the tumour thrombus in the IVC was not achieved in our cases with neoadjuvant sunitinib treatment. Although sunitinib treatment had noticeable effects on primary tumour size, metastases and survival, it had a limited effect on downsizing the extent of tumour thrombus in the IVC and did not affect the overall surgical outcome in this regard.

\section{Acknowledgements}

Publication: The results of the study were not published in full or in part in form of abstracts.

Contribution: There is not any other contributors who may not be listed as authors.

Conflict of Interest: No conflict of interest was declared by the authors.

Financial Disclosure: The authors declared that this study received no financial support.

\section{Ethics}

Informed Consent: Retrospective study.

Peer-review: Externally and internally peer-reviewed.

\section{Authorship Contributions}

Supervision: T.S., Concept: C.B., Design: T.F., C.B., Data Collection or Processing: A.B., Analysis or Interpretation: T.S., Literature Search: B.J., Writing: A.B., T.F., B.J.

\section{References}

1. Bigot P, Fardoun T, Bernhard J, et al. Neoadjuvant targeted molecular therapies in patients undergoing nephrectomy and inferior vena cava thrombectomy: is it useful? World J Urol 2014;32:109-114.

2. Borregales LD, Adibi M, Thomas AZ, et al. The role of neoadjuvant therapy in the management of locally advanced renal cell carcinoma. Ther Adv Urol 2016;8:130-141.

3. Cost N, Delacroix S Jr, Sleeper J, et al. The impact of targeted molecular therapies on the level of renal cell carcinoma vena caval tumor thrombus. Eur Urol 2011;59:912-918.

4. Pooleri GK, Nair TB, Sanjeevan KV, Thomas A. Neo adjuvant treatment with targeted molecules for renal cell cancer in current clinical practise. Indian J Surg Oncol 2012;3:114-119.

5. Schrader Al, Steffens S, Schnoeller T], et al. Neoadjuvant therapy of renal cell carcinoma: a novel treatment option in the era of targeted therapy? Int J Urol 2012;19:903-907.

6. Harshman LC, Srinivas S, Kamaya A, Chung BI. Laparoscopic radical nephrectomy after shrinkage of a caval tumor thrombus with sunitinib. Nat Rev Urol 2009;6:338-343.

7. Karakiewicz P, Suardi $N$, Jeldres $C$, et al. Neoadjuvant sutentinduction therapy may effectively down-stage renal cell carcinoma atrial thrombi. Eur Urol 2008;53:845-848.

8. Peters I, Winkler M, Juttner B, et al. Neoadjuvant targeted therapy in a primary metastasized renal cell cancer patient leads to downstaging of inferior vena cava thrombus (IVC) enabling a cardiopulmonary bypass-free tumor nephrectomy: a case report. World J Urol 2014;32:245-248.

9. Bex A, Van der Veldt AA, Blank C, et al. Progression of a caval vein thrombus in two patients with primary renal cell carcinoma on pretreatment with sunitinib. Acta Oncol 2010;49:520-523. 